\title{
Biomarkers and microsatellite instability analysis of curettings can predict the behavior of FIGO stage I endometrial endometrioid adenocarcinoma
}

Anita Steinbakk ${ }^{1,2}$, Anais Malpica ${ }^{3}$, Aida Slewa ${ }^{1}$, Ivar Skaland ${ }^{1}$, Einar Gudlaugsson ${ }^{1}$, Emiel AM Janssen ${ }^{1}$, Kjell Løvslett ${ }^{2}$, Bent Fiane ${ }^{2}$, Arnold Jan Kruse ${ }^{4}$, Weiwei Feng ${ }^{5}$, Yu Yinhua ${ }^{6}$ and Jan P Baak $^{1}$

${ }^{1}$ Department of Pathology, Stavanger University Hospital, Stavanger, Norway; ${ }^{2}$ Department of Gynaecology, Stavanger University Hospital, Stavanger, Norway; ${ }^{3}$ Department of Pathology and Gynecologic Oncology, The University of Texas M.D. Anderson Cancer Center, Houston, TX, USA; ${ }^{4}$ Department of Gynecology, Academic Medical Center, Maastricht, The Netherlands; ${ }^{5}$ Department of Gynecology, Obstetrics and Gynecology Hospital of Fudan University, Shanghai, People's Republic of China and ${ }^{6}$ Department of Experimental Medicine, The University of Texas M.D. Anderson Cancer Center, Houston, TX, USA

The prognostic value of molecular biomarkers, microsatellite instability, DNA ploidy and morphometric mean shortest nuclear axis in endometrial cancer is conflicting, possibly due to the fact that different studies have used mixtures of histotypes, FIGO stages and different non-standardized non-automated methods. We have evaluated the prognostic value of classical prognostic factors, molecular biomarkers, microsatellite instability, DNA ploidy and morphometric mean shortest nuclear axis in a population-based cohort of FIGO stage I endometrial endometrioid adenocarcinomas. Curettings of 224 FIGO stage I endometrial endometrioid adenocarcinoma patients were reviewed. Clinical information, including follow-up, was obtained from the patients' charts. Microsatellite instability and morphometric mean shortest nuclear axis were obtained in whole tissue sections and molecular biomarkers using tissue microarrays. DNA ploidy was analyzed by image cytometry. Univariate (Kaplan-Meier method) and multivariate (Cox model) survival analysis was performed. With median follow-up of 66 months (1-209), $14(6 \%)$ patients developed metastases. Age, microsatellite instability, molecular biomarkers (p16, p21, p27, p53 and survivin) and morphometric mean shortest nuclear axis had prognostic value. With multivariate analysis, combined survivin, p21 and microsatellite instability overshadowed all other variables. Patients in which any of these features had favorable values had an excellent prognosis, in contrast to those with either high survivin or low p21 (97 vs 78\% survival, $P<0.0001$, hazard ratio $=7.8$ ). Combined high survivin and low p21 values and microsatellite instability high identified a small subgroup with an especially poor prognosis (survival rate $57 \%, P=0.01$, hazard ratio $=5.6$ ). We conclude that low 221 and high survivin expression are poor prognosis indicators in FIGO stage I endometrial endometrioid adenocarcinoma, especially when high microsatellite instability occurs.

Modern Pathology (2011) 24, 1262-1271; doi:10.1038/modpathol.2011.75; published online 6 May 2011

Keywords: endometrial cancer; FIGO I; microsatellite instability; prognostic; p21; survivin

Correspondence: Professor JP Baak, MD, PhD FRCPath, FIAC(Hon), Department of Pathology, Stavanger University Hospital, Armauer Hansensvei 20, 4068, Stavanger, Norway. E-mail: jpabaak@yahoo.com

Received 25 November 2010; revised 9 March 2011; accepted 9 March 2011; published online 6 May 2011
Endometrial carcinoma is the most frequent gynecological cancer. The disease-related death rate in FIGO stage II-IV is high (20-80\% and higher), while in the 'favorable' early stage FIGO I, the death rate ranges from 5 to $15 \%,{ }^{1,2}$ which has been stable for decades. ${ }^{3}$ This prompts the search of other prognostic indicators to enable a more accurate triaging 
of patients concerning treatment modalities, and to gain a better understanding of the pathogenetic mechanisms in this disease.

In early FIGO stage I cancers, histological type, grade and myometrial invasion depth are often used to determine individual therapy, but their prognostic accuracy and reproducibility are not always optimal. ${ }^{2-4}$ More recently, nuclear morphometrical features (especially mean shortest nuclear axis, MSNA) and DNA ploidy were shown to be strongly prognostic in stage I types 1 and 2 cancers. ${ }^{3,5-8}$ An increased understanding of the molecular biology in endometrial carcinogenesis has revealed several promising diagnostic, prognostic and predictive biomarkers, such as microsatellite instability and hypermethylation. However, in many studies, a mix of early and late FIGO stage cancers of all histologic subtypes has been analyzed; the results of these studies may not be directly extrapolated to early cancers of the endometrioid type (which are by far the most common).

We previously found that in surgical-pathologically confirmed FIGO stage I-IIA endometrial endometrioid carcinomas, using the combination of Survivin, p21 and p53 biomarkers has a stronger prognostic value than classical parameters, either alone or combined. However, other biomarkers were not useful in these FIGO stage I endometrial endometrioid cases, in contrast to the reports of other investigators who analyzed a mixture of high and low stage endometrial cancers. ${ }^{9}$ Moreover, the independent prognostic value of microsatellite instability, mean shortest nuclear axis and DNA ploidy to the molecular biomarkers is also unknown. In the present study, we, therefore, ascertained the role of biomarkers, microsatellite instability, mean shortest nuclear axis and DNA ploidy analysis in predicting the behavior of FIGO stage I endometrial endometrioid cancer cases.

\section{Materials and methods}

Regional Ethics Committee and Norwegian Data Inspection approval was obtained before the initiation of this study. The cohort in this study (224 cases) is a population-based material from the South Rogaland county, Norway. The patients were selected from 363 cases of endometrial cancer, diagnosed between 1989 and 2004 at the Stavanger University Hospital. Twenty cases were excluded because of the lack of follow-up or no diagnostic evidence of invasive adenocarcinoma on histologic material reviewed by two gynecologic pathologists (EG and JPB) and 40 cases were excluded because of the lack of histologic material for additional studies. Surgery was performed shortly after the diagnosis of cancer on the curettage material. Patients considered having FIGO stage I or IIA cancers did not receive preoperative radiotherapy (RT); surgical treatment was total abdominal hysterectomy with bilateral salpingo-oophorectomy, but no lymphadenectomy or extensive staging. None of the patients were given preoperative hormonal treatment. Adjuvant postoperative RT was administered to all patients with FIGO I-C poorly differentiated endometrioid carcinomas or higher stage cancers. Curettings were fixed in $4 \%$ buffered formaldehyde, dehydrated, paraffin embedded, cut at $4 \mu \mathrm{m}$ and hematoxylin-eosin-saffran stained. The presence or absence of cervical and myometrium invasion was re-evaluated by two of us (EG and JPB) and the histologic grade and histotype were re-assessed by three independent gynecological pathologists (AM, EG and JPB) using the WHO 2003 classification. ${ }^{10}$ After this re-review, additional cases were excluded as follows: 24 cases of FIGO I-II non-endometrioid endometrial cancers, 6 cases of FIGO III-IV nonendometrioid cancers, 22 cases of endometrioid FIGO III-IV cancers and 27 cases of FIGO II cancers. Immunohistochemical studies were used as needed to ensure the accuracy of the histotype. A total of 224 cases of endometrial endometrioid adenocarcinoma FIGO stage I represent the cohort of the study.

\section{Biomarkers Studied and Methods}

The immunohistochemical biomarkers and methods have been described in detail elsewhere. ${ }^{9}$ The following biomarkers were assessed: p27 and p21 (cell-cycle regulators), p53 and p16 (tumor suppressors), p63 (apoptosis inducer and stem cell marker), cyclin E and Her-2 (proliferation-associated markers), survivin (apoptosis inhibitor), CK5/6 (differentiation marker) and PTEN/Akt. The best-preserved and least differentiated area of the tumor in each case was marked. Subsequently, this area was identified in the corresponding paraffin block to obtain two cylinders of $1.2 \mathrm{~mm}$, which were then inserted into a Tissue Micro Array block.

Antigen retrieval methods and antibody dilutions were optimized before the onset of the study. All sections were freshly cut and processed simultaneously as described before. ${ }^{9}$ Quantitative analysis of immunohistochemical expression was done, using tissue microarray cylinders with clearly different intensity and percentage positivity for each feature as control 'calibration' standards. The percentage of positive cells for p16, p21, p27, p53, p63 and CK $5 / 6$ was determined. Due to the specific staining pattern of survivin (scattered positive cell), the number of positive nuclei per $1.11 \mathrm{~mm}^{2}$ (ie the surface area of one tissue microarray cylinder at specimen level, after correction for irregular cylinder boundaries) was used as the 'Survivin Index.' The PTEN and Akt staining was evaluated as either positive or negative. The Her-2-stained sections were scored according to the FDA-approved scoring system provided by the manufacturer, as either $0,1+, 2+$ or $3+$. Careful quality control showed good reproducibility for each of the 
immunohistochemical variables studied (overall agreement between AS and JPB, 90\% and higher).

\section{Quantitative Image Analysis}

Nuclear morphometric analysis of the representative H\&E sections used for revision grading was performed with the motorized QPRODIT 6.1. image analysis system (Leica, Cambridge, UK) as described. ${ }^{8,11}$ For nuclear quantitative mean shortest nuclear axis assessments, the material was inadequate for 22 patients leaving 202 FIGO I patients for mean shortest nuclear axis analysis. As the present investigation is a validation study, we measured the mean shortest nuclear axis only, with the straightline-length module of QPRODIT at $\times 1800$ screen magnification, ${ }^{12}$ using rigid point-weighted systematic random sampling. ${ }^{13}$ This guarantees unbiased high reproducibility and stronger prognostic value. ${ }^{11}$ Intra- and inter-observer reproducibility of this method has previously proven to be very high. ${ }^{14}$

\section{Microsatellite and DNA Ploidy Analysis}

Microsatellite analysis was performed, as described before, using DNA isolated from archived, paraffinembedded tissue blocks, applying the QIAamp DNA Mini Kit (Qiagen ${ }^{\mathrm{TM}}$, Hilden, Germany) and the manufacturer's protocol for DNA isolation. In all, 53 patients were excluded from microsatellite instability analysis (too little/bad quality DNA material), leaving 171 patients for microsatellite instability analysis. Microsatellite instability analysis was performed with five markers (BAT-26, BAT-25, NR-21, NR-24 and NR-27) known to be quasimonomorphic and with low risk for polymorphisms in the Caucasian population, as previously described. ${ }^{15-18}$ The selected markers show a high sensitivity for microsatellite instability without the need for matching with patient's normal DNA. PCR amplification was performed under standard conditions. The product length was analyzed in a sequencer (GeneAnalyzer $^{\mathrm{TM}}$ 3130XL, Applied Biosystems) using the GeneMap ${ }^{\mathrm{TM}}$ software. Microsatellite instability in any marker was visualized as changes in the product length. Instability in $\geq 40 \%$ ( $\geq 2$ of 5 ) of the markers was regarded as high-frequency microsatellite instability, in 1 of 5 as low frequency and in no markers as microsatellite stable.

DNA ploidy analysis was performed on cell suspensions prepared from archived, paraffinembedded blocks, following European guidelines. ${ }^{19}$ Cytospins were Feulgen stained with pararosanilin under strictly standardized conditions and cytometric analysis was accomplished on a fully automated DNA image cytometer $\left(\mathrm{QPath}^{\mathrm{TM}}\right.$, Leica, Cambridge, UK). ${ }^{20}$ Eighty patients were excluded (too little material/bad quality material), leaving 144 FIGO I patients for ploidy analysis. At least 3000 (up to 5000) objects per slide were fully automatically scanned. Using predefined densitometric and geometric filters inclusion and measurement of fragments, non-epithelial cancer cells, inflammatory cells and cell clumps was avoided. The objects selected were visually inspected by an experienced cytologist/cytometrist and non-cancer cells were eliminated. At least 1000 objects should remain after the interactive cleaning procedure for further analysis. Diploid cancers were characterized as having a DNA index (DI) of $0.9-1.1$, while DI $<0.9$ or $\geq 1.1$ were regarded as aneuploid.

\section{Statistical Analysis}

We analyzed the following end points: alive with local or distant recurrence $(n=3)$, and dead of endometrial cancer $(n=11)$. As recurrence and dead of endometrial cancer gave the same results, any recurrence and dead of endometrial cancer cases were grouped together and further described. Both locoregional recurrences and metastases were included as 'recurrence,' but none of the patients had isolated lymph node metastases. Patients with death from other non-endometrial cancer-related causes, or patients lost to follow-up were censored at the last known follow-up date as alive, no evidence of disease. Analyses were performed by SPSS version 15 (SPSS, Chicago, IL, USA). Receiver operating curve (ROC) analysis (MedCalc Software, Mariakerke, Belgium) was also used, which means that the values/thresholds with the objectively best sensitivity and specificity were selected. ${ }^{21}$ Univariate analysis was performed using the Kaplan-Meier method, and differences in survival were estimated by the Breslow and log-rank tests. Multivariate survival analysis (Cox model) was used to assess the independent prognostic value of the different features. Hazard ratios and 95\% confidence intervals were calculated for each feature.

\section{Results}

The median age of the patients was 66 (range 37-94) years. With 66 months median follow-up (range, 1-209), 14 (6\%) patients had developed recurrent disease. Table 1 shows the correlation between microsatellite instability and ploidy. All microsatellite instability cases were diploid. All aneuploid

Table 1 The relation between MSS, MSI low and MSI high and ploidy

\begin{tabular}{lccc}
\hline & Diploid & Aneuploid & Total \\
\hline MSS & 96 & 14 & 110 \\
MSI low & 14 & 0 & 14 \\
MSI high & 20 & 0 & 20 \\
Total & 130 & 14 & 144 \\
\hline
\end{tabular}


cases were microsatellite stable, but many microsatellite stable cases $(96 / 110=87 \%)$ were diploid.

Table 2 summarizes the univariate prognostic value of the features analyzed. Age $(\leq 68$ vs $>68)$, many molecular biomarkers, particularly p21, p27, survivin, p53 and p16 (using optimal thresholds set by ROC analysis) and mean shortest nuclear axis ( $\leq 7.6$ vs $>7.6$ micrometer) were prognostic in this curettage material. We had $23 \%$ poorly differentiated cancers. In all, $21 \%$ of the TMA cylinders

Table 2 Survival data stratified for the different features analyzed

\begin{tabular}{|c|c|c|c|c|c|}
\hline Variable & Thresholds & Events/total number & $\%$ ANED & $\mathrm{P}$-value & $\begin{array}{c}H R \\
(95 \% C I)\end{array}$ \\
\hline \multirow[t]{2}{*}{ Age } & $\leq 68$ & $4 / 126$ & 97 & & \\
\hline & $>68$ & $10 / 98$ & & 0.004 & $\begin{array}{c}4.7 \\
(1.5-15.3)\end{array}$ \\
\hline \multirow[t]{2}{*}{ Type } & Endometrioid & $12 / 205$ & 90 & & \\
\hline & Endometrioid/ $<10 \%$ SPCA & $2 / 19$ & 90 & 0.40 & \\
\hline \multirow[t]{3}{*}{ Grade } & 1 & $1 / 38$ & 97 & & \\
\hline & 2 & $11 / 163$ & 93 & & \\
\hline & 3 & $2 / 23$ & 91 & 0.52 & \\
\hline \multirow{2}{*}{ Mean shortest nuclear axis } & $\leq 7.6$ & $12 / 193$ & 94 & & \\
\hline & $>7.6$ & $2 / 9$ & 78 & 0.03 & $\begin{array}{c}4.7 \\
(1.0-21.1)\end{array}$ \\
\hline \multirow[t]{2}{*}{ p16 } & $\leq 95 \%$ & $8 / 180$ & 96 & & \\
\hline & $>95 \%$ & $2 / 10$ & 80 & 0.02 & $\begin{array}{c}5.3 \\
(1.1-25.5)\end{array}$ \\
\hline \multirow[t]{2}{*}{$\mathrm{p} 21$} & $>1 \%$ & $9 / 186$ & 95 & & \\
\hline & $\leq 1 \%$ & $5 / 23$ & 78 & 0.002 & $\begin{array}{c}5.0 \\
(1.6-14.4)\end{array}$ \\
\hline \multirow[t]{2}{*}{$\mathrm{p} 27$} & $\geq 30 \%$ & $5 / 124$ & 96 & & \\
\hline & $<30 \%$ & $9 / 85$ & 89 & 0.05 & $\begin{array}{c}2.9 \\
(1.5-19.2)\end{array}$ \\
\hline \multirow[t]{2}{*}{ p53 } & $\leq 75 \%$ & $9 / 182$ & 95 & & \\
\hline & $>75 \%$ & $5 / 29$ & 83 & 0.02 & $\begin{array}{c}3.3 \\
(1.1-9.9)\end{array}$ \\
\hline \multirow[t]{2}{*}{ p63 } & $>1 \%$ & $8 / 143$ & 94 & & \\
\hline & $<=1 \%$ & $6 / 65$ & 91 & 0.29 & \\
\hline \multirow[t]{2}{*}{ CK5/6 } & $>6 \%$ & $4 / 78$ & 95 & & \\
\hline & $<=6 \%$ & $10 / 133$ & 93 & 0.52 & \\
\hline \multirow[t]{2}{*}{ Her-2 } & Neg & 8/139 & 94 & & \\
\hline & pos $($ Her2 $1+, 2+$ or $3+)$ & $6 / 73$ & 92 & 0.47 & \\
\hline \multirow[t]{2}{*}{ Cyclin E } & $\leq 25 \%$ & $4 / 70$ & 94 & & \\
\hline & $>25 \%$ & $10 / 141$ & 93 & 0.64 & \\
\hline \multirow[t]{2}{*}{ Survivin } & $\leq 158$ & $10 / 187$ & 95 & & \\
\hline & $>158$ & $4 / 21$ & 81 & 0.002 & $\begin{array}{c}5.0 \\
(1.6-16.1)\end{array}$ \\
\hline \multirow[t]{2}{*}{ PTEN } & Positive & $3 / 71$ & 96 & & \\
\hline & Negative & $7 / 119$ & 94 & 0.69 & \\
\hline \multirow[t]{2}{*}{ Akt } & Positive & $7 / 161$ & 96 & & \\
\hline & Negative & $3 / 33$ & 91 & 0.12 & \\
\hline \multirow[t]{2}{*}{ Ploidy } & Diploid & $6 / 130$ & 95 & & \\
\hline & aneuploid & $0 / 14$ & 100 & 0.37 & \\
\hline \multirow[t]{3}{*}{ MSI } & MSS & $6 / 125$ & 95 & & \\
\hline & MSI low & $0 / 19$ & 100 & & \\
\hline & MSI high & $4 / 27$ & 85 & 0.02 & $\begin{array}{c}4.6 \\
(1.3-16.7)\end{array}$ \\
\hline \multirow[t]{2}{*}{ MSI } & MSS/MSI low & $6 / 144$ & 96 & & \\
\hline & MSI high & $4 / 27$ & 85 & 0.005 & $\begin{array}{c}5.2 \\
(1.5-18.8)\end{array}$ \\
\hline \multirow[t]{2}{*}{ p21 and survivin combination } & $\mathrm{p} 21>1 \%$ and surviving $\leq 158$ & $5 / 163$ & 97 & & \\
\hline & All others & $9 / 42$ & 78 & $<0.0001$ & $\begin{array}{c}7.8 \\
(2.2-27.3)\end{array}$ \\
\hline \multirow[t]{2}{*}{ p21 > $1 \%$ and survivin $\leqslant 158$} & MSI low and MSS & $2 / 107$ & 98 & & \\
\hline & MSI high & $1 / 18$ & 94 & 0.16 & \\
\hline \multirow[t]{2}{*}{$\mathrm{p} 21 \leq 1 \%$ and/or survivin $>158$} & MSI low and MSS & $4 / 25$ & 84 & & \\
\hline & MSI high & $3 / 7$ & 57 & 0.01 & $\begin{array}{c}6.4 \\
(1.5-26.5)\end{array}$ \\
\hline
\end{tabular}

ANED, alive with no evidence of disease; HR, hazard ratio; CI, confidence interval; SPCA, serous papillary cancer; MSI, microsatellite instability; MSS, microsatellite stable; MSI low, microsatellite instability at low frequency; MSI high, microsatellite instability at high frequency; CK, cytokeratin. 
Table 3 Results of stepwise multivariate survival analysis (Cox model)

\begin{tabular}{|c|c|c|c|c|c|c|c|}
\hline & & \multirow[t]{2}{*}{$\beta$} & \multirow[t]{2}{*}{ Standard error } & \multirow[t]{2}{*}{ Sig. } & \multirow[t]{2}{*}{ Hazard ratio } & \multicolumn{2}{|c|}{$95 \%$ confidence interval for hazard ratic } \\
\hline & & & & & & Lower & Upper \\
\hline \multirow{3}{*}{$\begin{array}{l}\text { Step } 1 \\
\text { Step } 2\end{array}$} & $\mathrm{p} 21>\mathrm{vs} \leq 1 \%$ & 1.6 & 0.56 & 0.005 & 4.8 & 1.6 & 14.4 \\
\hline & $\mathrm{p} 21$ & 2.0 & 0.60 & 0.001 & 7.0 & 2.2 & 22.9 \\
\hline & Survivin $\leq v s>158$ & 2.0 & 0.64 & 0.001 & 7.8 & 2.2 & 27.3 \\
\hline Step 1 & Survivin $\leq v s>158$ & 1.9 & 0.69 & 0.005 & 7.2 & 1.8 & 28.1 \\
\hline \multirow[t]{2}{*}{ Step 2} & Survivin & 2.3 & 0.74 & 0.002 & 9.7 & 2.3 & 41.5 \\
\hline & $\mathrm{p} 21>v s \leq 1 \%$ & 1.5 & 0.73 & 0.038 & 4.5 & 1.1 & 18.9 \\
\hline \multirow[t]{3}{*}{ Step 3} & Survivin & 2.0 & 0.76 & 0.008 & 7.5 & 1.7 & 33.6 \\
\hline & p21 & 1.9 & 0.77 & 0.017 & 6.4 & 1.4 & 29.1 \\
\hline & MSI MSS \& low vs high & 1.7 & 0.71 & 0.016 & 5.6 & 1.4 & 22.4 \\
\hline
\end{tabular}

The variables entered are shown $(P$ to enter $<0.05)$. Top panel: results for p21 and survivin; bottom panel: p21, survivin and MSI. The difference between the significance of $\mathrm{p} 21$ and survivin is due to the fact that some cases have missing values for MSI.

were solid, the rest were glandular. The other $2 \%$ poorly differentiated cancers had $<50 \%$ solid areas but combined with severe atypia.

In this curettage material of type 1 endometrial cancers only, there was a (just not significant) trend $(P=0.08)$ that the well-differentiated cancers had a better prognosis than the moderately and poorly differentiated carcinomas. DNA ploidy was not prognostic either but very few cases were aneuploid in this endometrioid stage I endometrial cancer study. Microsatellite stable and low-frequency microsatellite instability cancers ( $84 \%$ of all cases) had a good prognosis, whereas high-frequency microsatellite instability cancers $(16 \%)$ were associated with a worse survival.

With multivariate analysis (Cox model, Forward Wald, probability to enter $<0.05$, total number of patients with all data available $=167)$, combined survivin $(P=0.008$, hazard ratio $=7.5,95 \%$ confidence interval $=1.7-33.6)$, p21 $(P=0.02$, hazard ratio $=6.4, \quad 95 \%$ confidence interval $=1.4-29.1)$ and microsatellite instability $(P=0.02$, hazard ratio $=5.6, \quad 95 \%$ confidence interval $=1.4-22.4$ ) overshadowed all other variables tested for prognostic value (Table 3). The prognostic strengths of p21 and survivin are nearly equivalent. The p21 was slightly stronger and selected first when only p21 and survivin were entered (Table 3). For many patients, microsatellite instability material was inadequate and data lacking. As a result, the patient numbers differed for p21 and survivin $(n=205)$, vs p21 and survivin and microsatellite instability entered $(n=167)$. In the latter analysis, survivin was slightly stronger and selected first, followed by p21 and as third variable microsatellite instability. Patients in which both p21 or survivin had favorable values (see Table 2 for the thresholds) had an excellent prognosis, contrasting those with unfavorable values of either survivin or p21 (survivin $>158$ or p211\%) (97 vs $78 \%$ survival, $P<0.0001$, hazard ratio $=7.8$; Figure 1). Combined p21/survivin did not correlate with grade or stage (FIGO Ia and Ib).

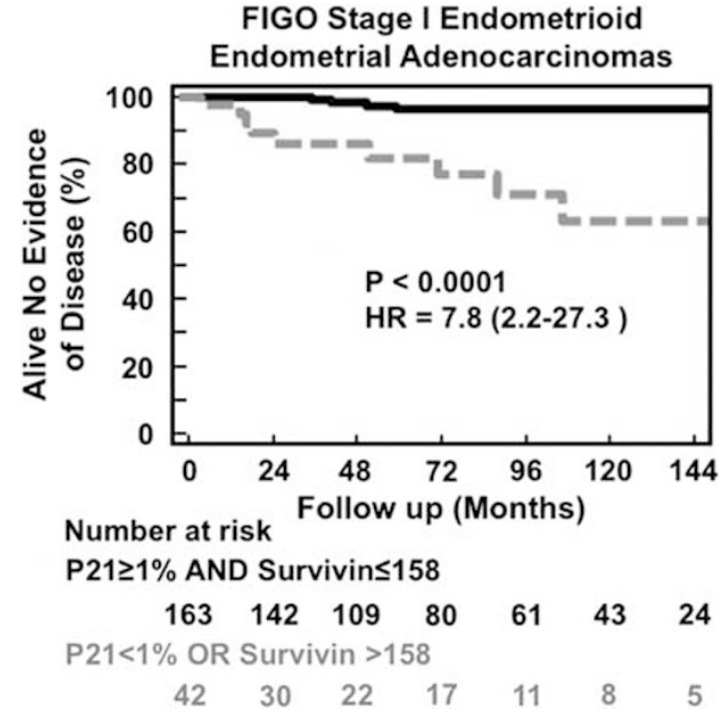

Figure 1 Survival curve of the FIGO I endometrioid endometrial cancer patients, stratified, according to p21 and survivin expression in the curettages.

In the group of patients with unfavorable high survivin or low p21 values $(n=42)$, high-frequency microsatellite instability identified a small subgroup $(n=7)$, with an especially poor prognosis (10-year survival rate of $57 \%, P=0.03$, hazard ratio $=5.6$ ). Figures 2 and 3 show examples of the combination of the histopathological images, p21 and survivin expression and microsatellite instability status in relation to prognosis.

\section{Discussion}

In this population-based FIGO stage I endometrial endometrioid cancer study, a combination of molecular quantitative biomarkers p21 and survivin has strongest prognostic value. Microsatellite instability contributes additional prognostic value to 

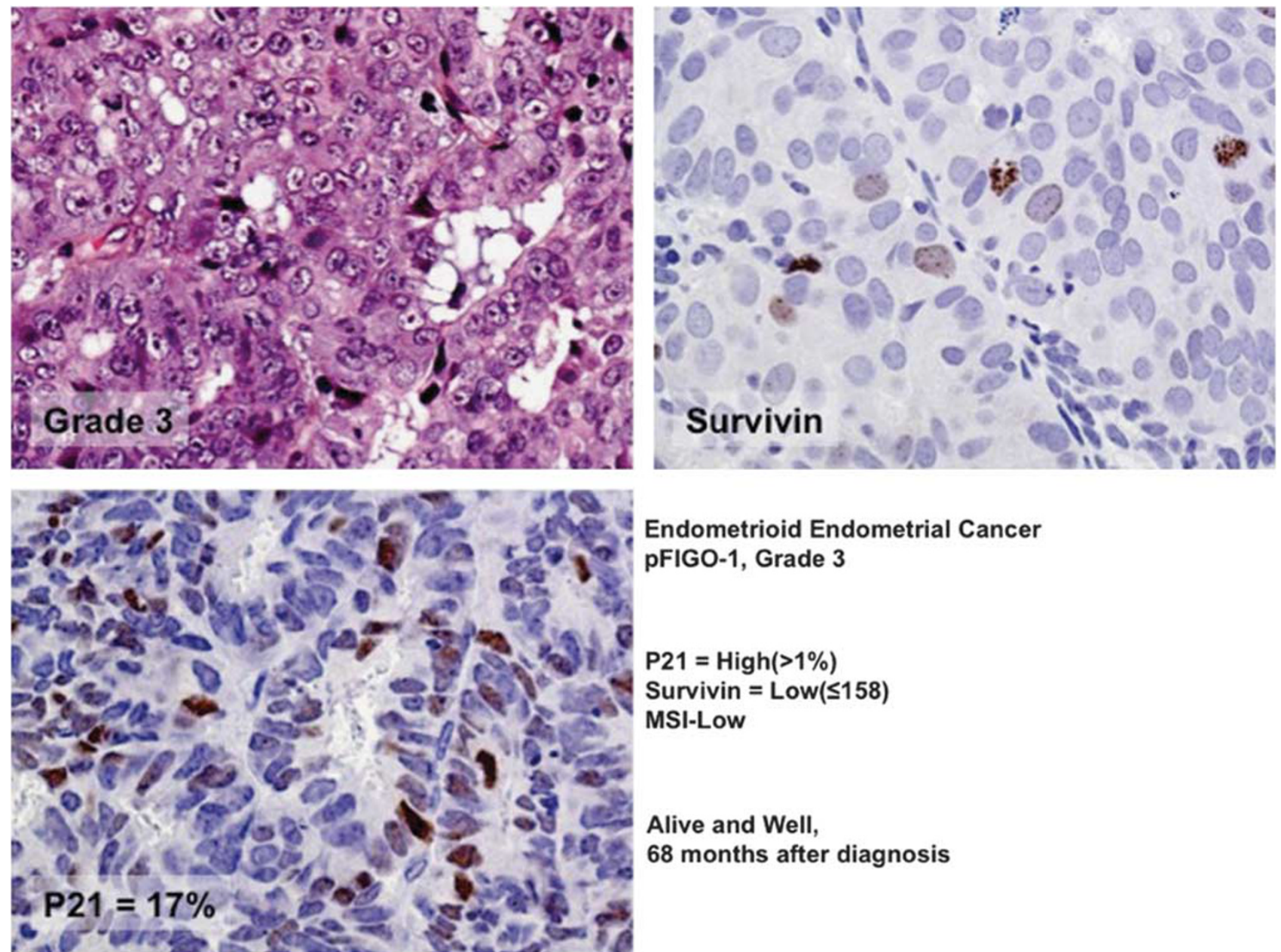

Figure 2 Favorable combination of biomarkers p21.

the patients with an unfavorable combination of any of these markers but not in those with favorable survivin/p21 phenotype. The strong prognostic value is consistent with their molecular biological functions, as survivin is localized at the mitotic spindle, binds caspase and can thus protect the cell from apoptosis. High values of survivin in endometrial cancer are, therefore, understandably unfavorable for the patient. p21 is a cyclin-dependent kinase inhibitor and an important cell-cycle downregulator, explaining why low expression will contribute to increased proliferation and hence poor prognosis.

A PubMed literature search for any of the biomarkers studied with the additional keywords 'endometrial cancer, immunohistochemical, prognostic' resulted in $>100$ studies, with impressive disagreement and variation regarding stage, histologic type, expression and prognostic value of the biomarkers. The major reasons for the differences seem small numbers of patients, mixtures of all stages and different histological types studied. Few studies have analyzed survivin in endometrial

\section{Endometrioid Endometrial Cancer} pFIGO-1, Grade 3

$\mathrm{P} 21=\mathrm{High}(>1 \%)$

Survivin $=\operatorname{Low}(\leq 158)$

MSI-Low

Alive and Well,

68 months after diagnosis cancer and all found overexpression to be unfavorable. In one study of 31 cases $^{22}$ high survivin was prognostic. Low p21 was prognostic in one other study. ${ }^{23}$ On the other hand, some biomarkers were not prognostic with multivariate analysis in our study contrasting other publications, but only very few of these were on endometrioid FIGO stage I cases. Examples are p53, prognostic in 9 out of 15 studies, but only one of these studies concerned exclusively FIGO stage I endometrioid cancers. ${ }^{24}$ Five of 11 studies found Her-2 to be of prognostic value, of which one, with a limited number of patients, found prognostic value in low-stage endometrioid cancers. ${ }^{25} \mathrm{P} 16$ was prognostic in two of nine previous studies, ${ }^{26,27}$ but these again analyzed mixtures of stages and subtypes. CK 5/6, prognostic in one study encompassing a mixture of stages and histotypes. ${ }^{28}$ PTEN was prognostic in four of eight studies. However, one was only for patients undergoing chemotherapy; in another there was no information about FIGO stage and histologic type, the third encompassed FIGO stage I cases, but mixed histologic types and the fourth used small numbers. ${ }^{29-32}$ 

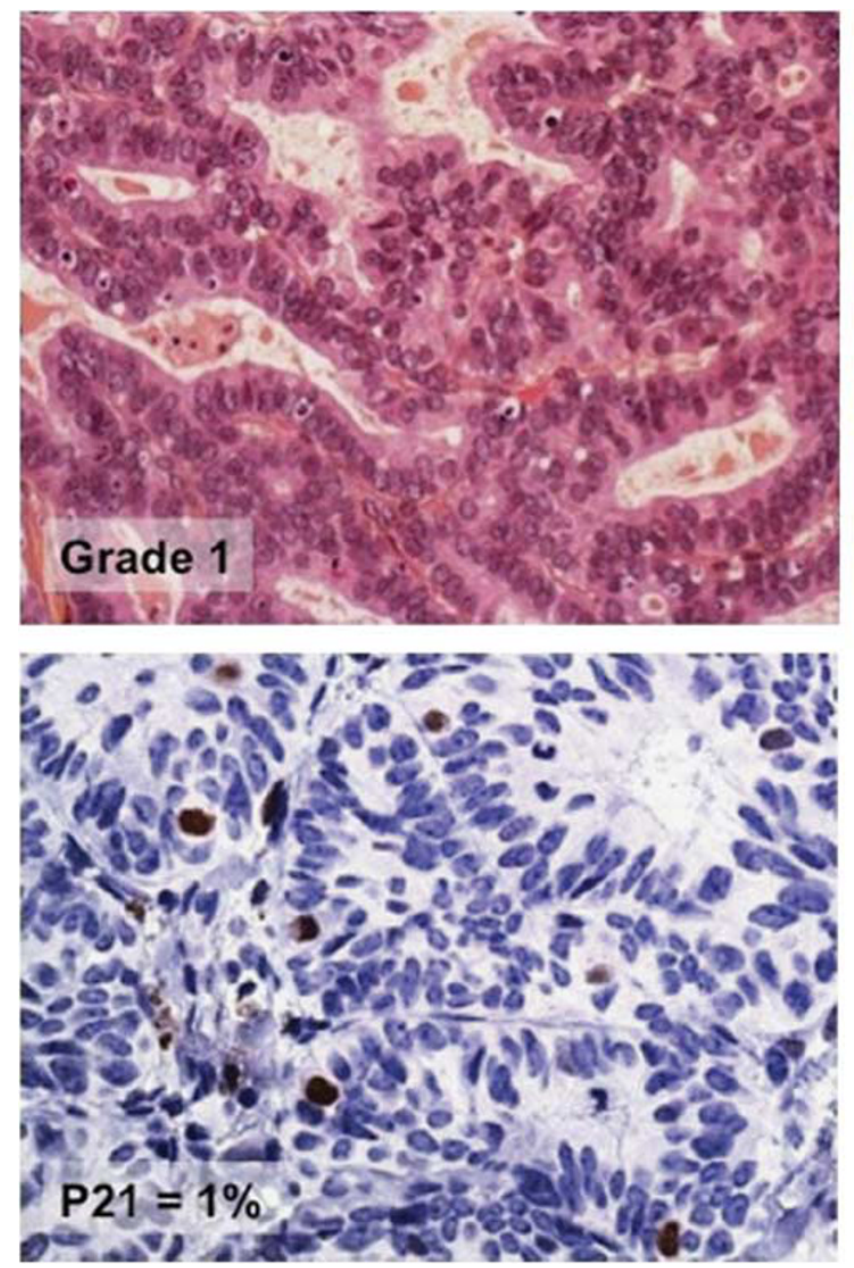

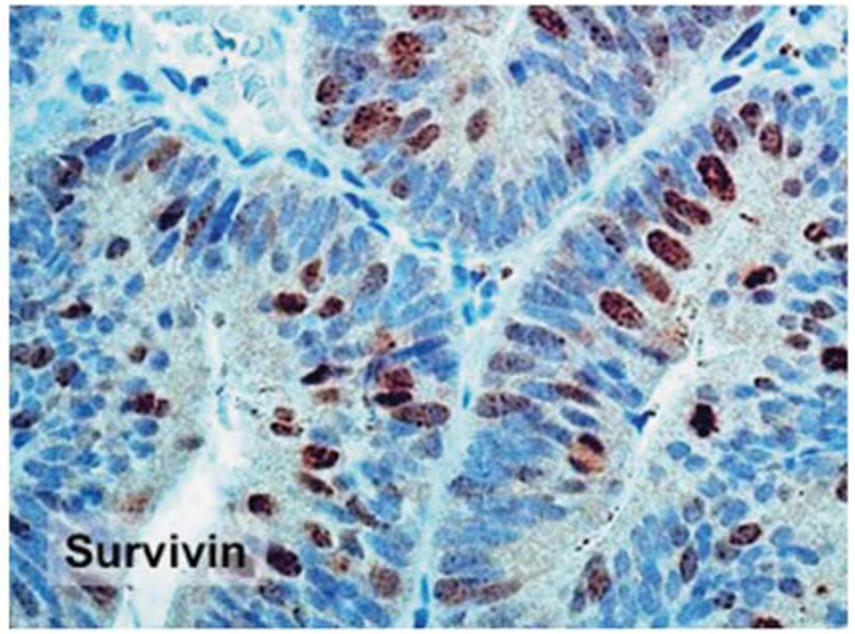

\section{Endometrioid Endometrial Cancer} pFIGO-1, Grade 1

P21 $=$ Low $(\leq 1 \%)$

Survivin $=$ High $(>158)$

MSI-High

\section{Dead of endometrial cancer}

Figure 3 Unfavorable combination of biomarkers p21, survivin and microsatellite instability.

In our study, p63 and cyclin E did not have prognostic value, in agreement with nearly all other studies on these biomarkers.

Data in the current literature regarding the prognostic significance of microsatellite instability in endometrial cancer are equally conflicting, with as many studies showing prognostic value as not. We found low-frequency microsatellite instability and high-frequency microsatellite instability in 11 and $16 \%$ of the cancers analyzed (in total $27 \%$ ). The prevalence of microsatellite instability in endometrial cancer in the literature shows a wide variation ranging from 9 to $45 \%,{ }^{33-46}$ which again may be due to the use of different combinations of endometrial cancers regarding type and stage, but also differences in molecular techniques used (especially in the older studies when there was less consensus about the definition of markers used). Combining different types and stages is likely to blur the results, as type II tumors have a lower frequency of microsatellite instability than type I, more often show aneuploidy and more aggressive behavior. This may also explain why DNA aneuploidy is so very rare in the current type 1 cancers and ploidy is not significant as a prognostic indicator in the present study. However, many (80) samples had to be excluded for technical reasons from our DNA ploidy analysis and the ploidy results, therefore, should be interpreted with care.

Grade was not significant, which can be surprising. However, the material consisted of curettings and not hysterectomies. Moreover, there was a (just not significant) trend $(P=0.08)$ that the well-differentiated cancers had a better prognosis than the moderately and poorly differentiated carcinomas. Finally, it should be remembered that the material consisted of type 1 cancers only. Other studies often use mixtures of type 1 and 2 cancers. Type 2 cancers often are moderately or poorly differentiated.

The morphometric feature mean shortest nuclear axis previously was strongly prognostic in a mixture of all histologic type endometrial cancers. In the current study on FIGO I type 1 cancers only, mean shortest nuclear axis was overshadowed by the molecular biomarkers. Interestingly, mean shortest nuclear axis was prognostically stronger than grade, possibly due to the objective quantitative nature of the morphometric feature. 


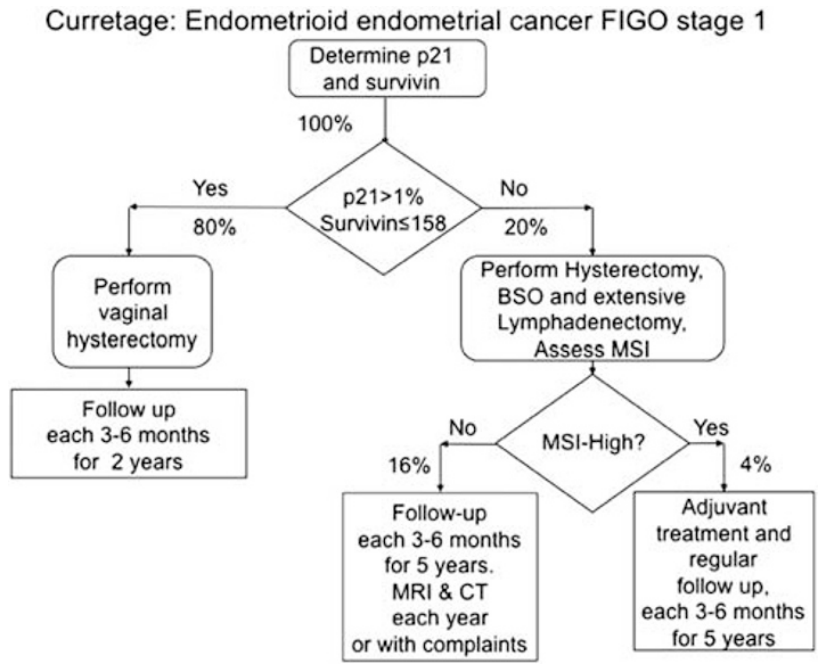

Figure 4 Therapeutic decision algorithm based on histologic type and molecular biomarkers assessed in curettage material. BSO, bilateral salpingo-oophorectomy.

Another important question is the significance of hypermethylation in FIGO stage I endometrioid endometrial cancer. Type 1 cancers often show K-ras and Braf mutations, and the current article shows that high-frequency microsatellite instability is not infrequent. In colorectal cancer, highfrequency microsatellite instability is related to hypermethylation. Promoter hypermethylation of certain genes appears to be an early and frequent event in endometrial carcinogenesis. ${ }^{47,48}$ Type I cancers often show K-ras mutations, which are correlated with microsatellite instability. ${ }^{49}$

As our aim was to find prognostic markers for early stage endometrioid endometrial cancer in curettage material, we had to evaluate FIGO IA and IB as one group; it is difficult/impossible to separate in curettage material FIGO stage IA and B with certainty.

Upregulation of the apoptosis inhibitor survivin and downregulation of cell-cycle suppressor p21 appear to be early prognostic biomarkers in endometrioid endometrial cancer, as many cases displayed high survivin and low p21 without developing metastatic disease. On the other hand, high-frequency microsatellite instability is associated with very high recurrence if survivin or p21 are unfavorable. It would be interesting to analyze these biomarkers in endometrial precancers (endometrial intraepithelial neoplasia).

The sharp increase of endometrial cancer incidence in the Western world during the last few decades, including younger women in childbearing age, raises the important point of fertility-sparing and ovary-sparing surgery. ${ }^{50}$ Patients with obesity, diabetes or cardiovascular disease among others, also would benefit from less aggressive surgery such as vaginal hysterectomy and no lymphadenectomy. Survivin and p21 can easily and reliably be assessed by immunohistochemistry in preoperative curettage and could in principle well be used in routine clinical practice for tailoring patient management to determine whether a patient should have surgery with or without node sampling For low-risk patients, or patients with an increased operation risk mentioned above, less aggressive management with a vaginal approach to surgery may be sufficient, alleviating suffering and reducing health-care costs. On the other hand, in patients with p21/ survivin high-risk characteristics (about $20 \%$ of all FIGO I endometrial endometrioid cancers), additional microsatellite instability analysis could detect patients with an especially poor prognosis, who may be considered for aggressive adjuvant chemotherapy. Figure 4 summarizes these therapeutic implications of the molecular biomarkers.

As far as we know, our population-based endometrioid FIGO I endometrial cancer study is the largest with long and complete follow-up. We conclude that in FIGO stage I, endometrial endometrioid cancer curettages, survivin, p21 and microsatellite instability have strong independent prognostic value. Validation studies are required to confirm these results.

\section{Acknowledgements}

Anita Steinbakk is a PhD student from Helse Vest, Grant \#911268. This work has also been supported by Grant 09-1252 of the Stichting Bevordering Diagnostische Morfometrie, Middelburg, The Netherlands.

\section{Disclosure/conflict of interest}

The authors declare no conflict of interest.

\section{References}

1 Creasman W. Revised FIGO staging for carcinoma of the endometrium. Int J Gynaecol Obstet 2009;105:109.

2 Baak JP, Snijders W, van Diermen B, et al. Prospective multicenter validation confirms the prognostic superiority of the endometrial carcinoma prognostic index in international Federation of gynecology and obstetrics stage 1 and 2 endometrial carcinoma. J Clin Oncol 2003;21:4214-4221.

3 Van der Putten HW, Baak JP, Koenders TJ, et al. Prognostic value of quantitative pathologic features and DNA content in individual patients with stage I endometrial adenocarcinoma. Cancer 1989;63: 1378-1387.

4 Zaino RJ, Silverberg SG, Norris HJ, et al. The prognostic value of nuclear versus architectural grading in endometrial adenocarcinoma: a Gynecologic Oncology Group study. Int J Gynecol Pathol 1994;13:29-36.

5 Lundgren C, Auer G, Frankendal B, et al. Nuclear DNA content, proliferative activity, and p53 expression related to clinical and histopathologic features in endometrial carcinoma. Int J Gynecol Cancer 2002;12: 110-118. 
6 Salvesen HB, Iversen OE, Akslen LA. Prognostic impact of morphometric nuclear grade of endometrial carcinoma. Cancer 1998;83:956-964.

7 Sorbe B, Risberg B, Thornthwaite J. Nuclear morphometry and DNA flow cytometry as prognostic methods for endometrial carcinoma. Int J Gynecol Cancer 1994;4:94-100.

8 Baak JP, Snijders WP, Van Diest PJ, et al. Confirmation of the prognostic value of the ECPI-1 score (myometrial invasion, DNA-ploidy and mean shortest nuclear axis) in FIGO stage I endometrial cancer patients with long follow-up. Int J Gynecol Cancer 1995;5:112-116.

9 Steinbakk A, Skaland I, Gudlaugsson E, et al. The prognostic value of molecular biomarkers in tissue removed by curettage from FIGO stage 1 and 2 endometrioid type endometrial cancer. Am J Obstet Gynecol 2009;200:78 e1-8.

10 Silverberg SG, Kurman RJ, Nogales F, et al. Tumours of the uterine corpus In: Tavassoli FA, Devilee P (eds). Pathology and Genetics of Tumours of the Breast and Female Genital Organs. IARC Press: Lyon, 2003, pp 217-228, 219 pp.

11 Jannink I, Bennen JN, Blaauw J, et al. At convenience and systematic random sampling: effects on the prognostic value of nuclear area assessments in breast cancer patients. Breast Cancer Res Treat 1995;36: 55-60.

12 Fleege JC, Baak JP, Smeulders AW. Analysis of measuring system parameters that influence reproducibility of morphometric assessments with a graphic tablet. Hum Pathol 1988;19:513-517.

13 Sorensen FB, Ottosen PD. Stereological estimation of nuclear volume in benign and malignant melanocytic lesions of the skin. Inter- and intraobserver variability of malignancy grading. Am J Dermatopathol 1991; 13:99-107.

14 Mutter GL, Baak JP, Crum CP, et al. Endometrial precancer diagnosis by histopathology, clonal analysis, and computerized morphometry. J Pathol 2000;190: $462-469$.

15 Soreide K, Slewa A, Stokkeland PJ, et al. Microsatellite instability and DNA ploidy in colorectal cancer: potential implications for patients undergoing systematic surveillance after resection. Cancer 2009;115: 271-282.

16 Suraweera N, Duval A, Reperant M, et al. Evaluation of tumor microsatellite instability using five quasimonomorphic mononucleotide repeats and pentaplex PCR. Gastroenterology 2002;123:1804-1811.

17 Buhard O, Suraweera N, Lectard A, et al. Quasimonomorphic mononucleotide repeats for high-level microsatellite instability analysis. Dis Markers 2004;20: 251-257.

18 Buhard O, Cattaneo F, Wong YF, et al. Multipopulation analysis of polymorphisms in five mononucleotide repeats used to determine the microsatellite instability status of human tumors. J Clin Oncol 2006;24:241-251.

19 Haroske G, Baak JP, Danielsen H, et al. Fourth updated ESACP consensus report on diagnostic DNA image cytometry. Anal Cell Pathol 2001;23:89-95.

20 Baak JP, Bol MG, van Diermen B, et al. DNA cytometric features in biopsies of TaT1 urothelial cell cancer predict recurrence and stage progression more accurately than stage, grade, or treatment modality. Urology 2003;61:1266-1272.

21 Korner H, Soreide K, Stokkeland PJ, et al. Diagnostic accuracy of serum-carcinoembryonic antigen in recurrent colorectal cancer: a receiver operating characteristic curve analysis. Ann Surg Oncol 2007; 14:417-423.

22 Takai N, Miyazaki T, Nishida M, et al. Survivin expression correlates with clinical stage, histological grade, invasive behavior and survival rate in endometrial carcinoma. Cancer Lett 2002;184:105-116.

23 Salvesen HB, Iversen OE, Akslen LA. Prognostic significance of angiogenesis and Ki-67, p53, and p21 expression: a population-based endometrial carcinoma study. J Clin Oncol 1999;17:1382-1390.

24 Ito K, Watanabe K, Nasim S, et al. Prognostic significance of p53 overexpression in endometrial cancer. Cancer Res 1994;54:4667-4670.

25 Nazeer T, Ballouk F, Malfetano JH, et al. Multivariate survival analysis of clinicopathologic features in surgical stage I endometrioid carcinoma including analysis of HER-2/neu expression. Am J Obstet Gynecol 1995;173:1829-1834.

26 Salvesen HB, Das S, Akslen LA. Loss of nuclear p16 protein expression is not associated with promoter methylation but defines a subgroup of aggressive endometrial carcinomas with poor prognosis. Clin Cancer Res 2000;6:153-159.

27 Engelsen IB, Stefansson I, Akslen LA, et al. Pathologic expression of p53 or p16 in preoperative curettage specimens identifies high-risk endometrial carcinomas. Am J Obstet Gynecol 2006;195:979-986.

28 Stefansson IM, Salvesen HB, Akslen LA. Loss of p63 and cytokeratin 5/6 expression is associated with more aggressive tumors in endometrial carcinoma patients. Int J Cancer 2006;118:1227-1233.

29 Terakawa N, Kanamori Y, Yoshida S. Loss of PTEN expression followed by Akt phosphorylation is a poor prognostic factor for patients with endometrial cancer. Endocr Relat Cancer 2003;10:203-208.

30 Uegaki K, Kanamori Y, Kigawa J, et al. PTEN-positive and phosphorylated-Akt-negative expression is a predictor of survival for patients with advanced endometrial carcinoma. Oncol Rep 2005;14:389-392.

31 Athanassiadou P, Athanassiades P, Grapsa D, et al. The prognostic value of PTEN, p53, and beta-catenin in endometrial carcinoma: a prospective immunocytochemical study. Int J Gynecol Cancer 2007;17:697-704.

32 Erkanli S, Kayaselcuk F, Kuscu E, et al. Expression of survivin, PTEN and p27 in normal, hyperplastic, and carcinomatous endometrium. Int J Gynecol Cancer 2006;16:1412-1418.

33 Peiro G, Diebold J, Lohse P, et al. Microsatellite instability, loss of heterozygosity, and loss of hMLH1 and hMSH2 protein expression in endometrial carcinoma. Hum Pathol 2002;33:347-354.

34 Fiumicino S, Ercoli A, Ferrandina G, et al. Microsatellite instability is an independent indicator of recurrence in sporadic stage I-II endometrial adenocarcinoma. J Clin Oncol 2001;19:1008-1014.

35 Honore LH, Hanson J, Andrew SE. Microsatellite instability in endometrioid endometrial carcinoma: correlation with clinically relevant pathologic variables. Int J Gynecol Cancer 2006;16:1386-1392.

36 Wong YF, Ip TY, Chung TK, et al. Clinical and pathologic significance of microsatellite instability in endometrial cancer. Int J Gynecol Cancer 1999;9:406-410.

37 Black D, Soslow RA, Levine DA, et al. Clinicopathologic significance of defective DNA mismatch repair in endometrial carcinoma. J Clin Oncol 2006;24: 1745-1753. 
38 Peiro G, Lohse P, Mayr D, et al. Insulin-like growth factor-I receptor and PTEN protein expression in endometrial carcinoma. Correlation with bax and bcl-2 expression, microsatellite instability status, and outcome. Am J Clin Pathol 2003;120:78-85.

39 An HJ, Kim KI, Kim JY, et al. Microsatellite instability in endometrioid type endometrial adenocarcinoma is associated with poor prognostic indicators. Am J Surg Pathol 2007;31:846-853.

40 MacDonald ND, Salvesen HB, Ryan A, et al. Frequency and prognostic impact of microsatellite instability in a large population-based study of endometrial carcinomas. Cancer Res 2000;60:1750-1752.

41 Zighelboim I, Goodfellow PJ, Gao F, et al. Microsatellite instability and epigenetic inactivation of MLH1 and outcome of patients with endometrial carcinomas of the endometrioid type. J Clin Oncol 2007;25:2042-2048.

42 Muresu R, Sini MC, Cossu A, et al. Chromosomal abnormalities and microsatellite instability in sporadic endometrial cancer. Eur J Cancer 2002;38:1802-1809.

43 Hirasawa A, Aoki D, Inoue J, et al. Unfavorable prognostic factors associated with high frequency of microsatellite instability and comparative genomic hybridization analysis in endometrial cancer. Clin Cancer Res 2003;9:5675-5682.
44 Ju W, Park HM, Lee SN, et al. Loss of hMLH1 expression is associated with less aggressive clinicopathological features in sporadic endometrioid endometrial adenocarcinoma. J Obstet Gynaecol Res 2006;32: 454-460.

45 Maxwell GL, Risinger JI, Alvarez AA, et al. Favorable survival associated with microsatellite instability in endometrioid endometrial cancers. Obstet Gynecol 2001;97:417-422.

46 Orbo A, Eklo K, Kopp M. A semiautomated test for microsatellite instability and its significance for the prognosis of sporadic endometrial cancer in northern Norway. Int J Gynecol Pathol 2002;21:27-33.

47 Ignatov A, Bischoff J, Ignatov T, et al. APC promoter hypermethylation is an early event in endometrial tumorigenesis. Cancer Sci 2010;101:321-327.

48 Huang YW, Luo J, Weng YI, et al. Promoter hypermethylation of CIDEA, HAAO and RXFP3 associated with microsatellite instability in endometrial carcinomas. Gynecol Oncol 2010;117:239-247.

49 Lagarda H, Catasus L, Arguelles R, et al. K-ras mutations in endometrial carcinomas with microsatellite instability. J Pathol 2001;193:193-199.

50 Ries L, Melbert D, Krapcho M. SEER Cancer Statistics Review, 1975-2005. National Cancer Institute. 